\title{
A NOTE ON THE TEMPERATURE DEPENDENCE OF THE NORMAL STRESS MODULI
}

\author{
K. R. RAJAGOPAL and A. S. WINEMAN \\ Department of Mechanical Engineering and Applied Mechanics. The University of Michigan, Ann Arbor, \\ MI 48109, U.S.A.
}

(Communicated by T. W. TING)

\begin{abstract}
In this analysis we establish necessary and sufficient conditions which the normal stress modulus $\alpha_{1}(\theta)$ and its derivative $\mathrm{d} \alpha_{1}(\theta) / \mathrm{d} \theta$ ought to satisfy if a homogeneous incompressible second grade fluid is to meet the requirement that the specific internal energy of the fluid be a minimum when the fluid is locally at rest. We also require that all arbitrary motions of the fluid meet the Clausius-Duhem inequality. It is found that requiring that the specific internal energy of the fluid be a minimum when the fluid is locally at rest is not equivalent to a similar requirement on the specific Helmholtz free energy.
\end{abstract}

\section{INTRODUCTION}

A DETAILED study of the thermodynamics of an incompressible homogeneous fluid of second grade has been carried out by Dunn and Fosdick[1]. In that analysis, they show that if the fluid is to meet the restrictions imposed by the Clausius-Duhem inequality for all arbitrary motions, then the material moduli which characterize the fluid have to satisfy

$$
\mu(\theta) \geq 0, \quad \alpha_{1}(\theta)+\alpha_{2}(\theta)=0,
$$

and $\dagger$ the specific Helmholtz free energy is of the form

$$
\psi(\mathbf{x}, t)=\bar{\psi}\left(\theta, \mathbf{A}_{1}\right)=\bar{\psi}(\theta, 0)+\frac{\alpha_{1}(\theta)}{4 \rho}\left|\mathbf{A}_{1}\right|^{2} .
$$

$\mu$ represents the viscosity, $\alpha_{1}$ and $\alpha_{2}$ are the normal stress moduli, all considered functions of temperature. They further show that if one assumes that the specific Helmholtz free energy function is a minimum when the fluid is locally at rest, $\ddagger$ then

$$
\alpha_{1}(\theta) \geq 0 .
$$

The signs of the normal stress moduli $\alpha_{1}$ and $\alpha_{2}$ have been the subject matter of much controversy, a detailed account of which is provided in [1]. It involves the work of Ting[3], Coleman and Markovitz[4], Coleman, Duffin and Mizel[5], Coleman and Mizel[6] and Truesdell [7].

Assuming that the material moduli are independent of temperature and meet the restrictions imposed by eqns (1) and (2), Dunn and Fosdick [1] showed that a very general class of motions of an incompressible second grade fluid are stable in the sense that the spatially averaged kinetic energy and the energy due to stretching decay away in time. More recently, Fosdick and Rajagopal [8] established that these fluids exhibit certain anomalous characteristics if $\alpha_{1} \leq 0$, irrespective of what the sum of the material moduli $\left(\alpha_{1}+\alpha_{2}\right)$ is. However, these results have been established under the assumption that the material moduli are constant, and it could yet be possible that the fluid is stable

$\dagger$ It has been shown in [2], that for Rivlin-Ericksen fluids of grade $n$, if the specific Helmholtz free energy $\psi$ is assumred to be a function of the temperature, the temperature gradient and the first $n$ velocity gradients, then $\psi$ must actually be independent of the temperature gradient and the $n$th velocity gradient, i.e.

$$
\psi(\mathbf{x}, t)=\hat{\psi}\left(\theta, \mathbf{g}, \mathbf{A}_{1}, \ldots \mathbf{A}_{n}\right)=\bar{\psi}\left(\theta, \mathbf{A}_{1}, \ldots \mathbf{A}_{n-1}\right),
$$

where $g$ is the temperature gradient and $A_{n}$ are the first $n$ Rivlin-Ericksen tensors.

$\ddagger$ By this we mean $\bar{\psi}(\theta, 0) \leq \bar{\psi}\left(\theta, \mathbf{A}_{1}\right)$ for all traceless $\mathbf{A}_{1}$. 
to disturbances to the null flow if $\alpha_{1}$ is temperature dependent while being non-positive, i.e. $\alpha_{1}(\theta) \leq 0 . \dagger$

While it is well known that viscosity decreases with increasing temperature for fluids, knowledge about the variation of the normal stress coefficients $\alpha_{1}$ and $\alpha_{2}$ with respect to temperature appears to be limited from experimental observations. $\ddagger$ In this analysis we obtain certain necessary and sufficient conditions on $\alpha_{1}(\theta)$ and $\mathrm{d} \alpha_{1}(\theta) / \mathrm{d} \theta$, if the second grade fluid is to meet the requirement that the specific internal energy $\S$ of the fluid be a minimum when the fluid is locally at rest.

In Section 2, after a brief introduction regarding fluids of second grade, we derive a thermodynamic equality. In Section 3, we analyze the implications of the equality derived in the earlier section. We conclude this work with a few explanatory remarks in Section 4.

\section{FLUID OF GRADE TWO}

The Cauchy stress $\mathbf{T}$ in an incompressible homogeneous second grade fluid is related to the fluid motion in the following manner[9]

$$
\mathbf{T}=-p \mathbf{1}+\mu(\theta) \mathbf{A}_{1}+\alpha_{1}(\theta) \mathbf{A}_{2}+\alpha_{2}(\theta) \mathbf{A}_{1}{ }^{2},
$$

where $\mu(\theta)$ is the viscosity, $\alpha_{1}(\theta)$ and $\alpha_{2}(\theta)$ are the normal stress moduli. The $-p 1$ denotes the constitutively indeterminate spherical stress due to the constraint of incompressibility, and $\mathbf{A}_{1}$ and $\mathbf{A}_{2}$ are the first two Rivlin-Ericksen tensors defined through

$$
A_{1}=(\operatorname{grad} v)+(\operatorname{grad} v)^{T},
$$

and

$$
\mathbf{A}_{2}=\dot{\mathbf{A}}_{1}+\mathbf{A}_{1}(\operatorname{grad} \mathbf{v})+(\operatorname{grad} \mathbf{v})^{T} \mathbf{A}_{1} .
$$

In the above equations $\mathbf{v}$ denotes the velocity and the dot in eqn $(4)_{2}$ denotes material time differentiation.

Firstly, we derive a thermodynamic equality for fluids of second grade which will be useful in determining the variations of the normal stress moduli with temperature. It has been shown in [1], that, if a fluid of second grade is to undergo motions which meet the restrictions imposed by the Clausius-Duhem inequality, then, the specific entropy and the specific Helmholt free energy are related through the expression

$$
\eta\left(\theta, \mathbf{A}_{1}\right)=-\bar{\psi}_{\theta}\left(\theta, \mathbf{A}_{1}\right)
$$

In the above equation, $\bar{\psi}_{\theta}$ denotes the partial derivative of $\bar{\psi}$ with respect to first place. Since the specific Helmholtz free energy is related to the specific internal energy and the specific entropy through

$$
\psi \equiv \epsilon-\theta \eta
$$

it follows from eqns $(1)_{2}$ and (5), and the definition (6) that

$$
\bar{\epsilon}\left(\theta, \mathbf{A}_{1}\right)=\bar{\psi}(\theta, 0)-\theta \overline{\psi_{\theta}}(\theta, 0)+\frac{\alpha_{1}(\theta)}{4 \rho}\left|\mathbf{A}_{1}\right|^{2}-\frac{1}{4 \rho} \theta \frac{\mathrm{d} \alpha_{1}(\theta)}{\mathrm{d} \theta}\left|\mathbf{A}_{1}\right|^{2} .
$$

tThe condition $\alpha_{1}(\theta) \leq 0$, by virtue of eqn (1) contradicts the assumption that the specific Helmholtz free energy of the fluid be a minimum when the fluid is locally at rest. In fact the condition $\alpha_{1}(\theta) \leq 0$ implies the specific Helmholtz free energy is a maximum when the fluid is locally at rest. The condition that $\alpha_{1}(\theta) \leq 0$, however, does not contradict the Clausius-Duhem inequality.

fOne might reason that, since the normal stress moduli represent the deviation of the particular class of second grade fluids from a Newtonian fluid, and since increase in temperature seems to mitigate the non-Newtonian behavior of such fluids, the effect of the normal stress moduli ought to decrease with temperature.

\$Requiring that the specific internal energy of the fluid be a minimum when the fluid is locally at rest is not equivalent to a similar requirement on the specific Helmholtz free energy (see Section 3). 
Making use of the fact that

$$
\bar{\epsilon}(\theta, 0)=\bar{\psi}(\theta, 0)-\theta \bar{\psi}_{\theta}(\theta, 0)
$$

we find that eqn (7) yields

$$
\overline{\boldsymbol{\epsilon}}\left(\theta, \mathbf{A}_{1}\right)=\overline{\boldsymbol{\epsilon}}(\theta, 0)+\frac{\alpha_{1}(\theta)}{4 \rho}\left|\mathbf{A}_{1}\right|^{2}-\frac{1}{4 \rho} \theta \frac{\mathrm{d} \alpha_{1}(\theta)}{\mathrm{d} \theta}|\mathbf{A}|^{2} .
$$

Equation (9) will form the starting point for our analysis.

\section{ANALYSIS}

Firstly, we observe from the form of the free energy function (eqn $\left.(1)_{2}\right)$, that, if the normal stress modulus $\alpha_{1}$ is independent of temperature, then

$$
\tilde{\eta}\left(\theta, \mathbf{A}_{1}\right)=-\bar{\psi}_{\theta}\left(\theta, \mathbf{A}_{1}\right)=-\bar{\psi}_{\theta}(\theta, 0)
$$

Hence the following.

\section{Assertion 1}

If a homogeneous incompressible fluid of second grade undergoes a motion that meets the Clausius-Duhem inequality, and if the material moduli which characterize the fluid are independent of temperature, then the specific entropy of the fluid is a function of temperature only, i.e.

$$
\eta=\hat{\eta}(\theta) \text {. }
$$

\section{Remark}

Thus, requiring the material moduli to be independent of temperature implies that the specific entropy of the fluid is unaffected by variations in the kinematical Rivlin-Ericksen tensor $A_{1}$. Of course, the motion of the body does have an effect on the specific entropy, as the temperature $\theta$ is dependent on the motion.

Next, we would like to make a few observations on the now usual procedure of assuming that the specific Helmhiotz free energy be a minimum when the fluid is in equilibrium. The notion of Helmholtz free energy is a derived notion in thermodynamics, derived from the primitive ideas of specific internal energy and specific entropy. The assumption that it ought to be a minimum in equilibrium stems from the physical expectation that the specific internal energy be a minimum in equilibrium and the specific entropy be a maximum in equilibrium. The assumptions on the specific internal energy and the specific entropy and the relationship (6) then imply the assumption regarding the specific Helmholtz free energy. Firstly, let us investigate the consequences of the assumption that the specific internal energy of the fluid be a minimum when the fluid is locally at rest. We shall see that this assumption leads to the following interesting results on the nature of the normal stress moduli $\alpha_{1}(\theta)$.

\section{Assumption $1 \dagger$}

The specific internal energy of the fluid is a minimum when the fluid is locally at rest, i.e.

$$
\bar{\epsilon}(\theta, 0) \leq \bar{\epsilon}\left(\theta, \mathbf{A}_{1}\right)
$$

for all tensors $\mathbf{A}_{1}$ which are traceless.

$\dagger A$ weaker and more appropriate assumption would be to the effect that the global internal energy of the fluid be a minimum when the fuid is locally at rest, i.e.

$$
\int_{\Omega} \rho \bar{\epsilon}\left(\theta, \mathbf{A}_{1}\right) \mathrm{d} v \geq \int_{\Omega} \rho \bar{\epsilon}(\theta, 0) \mathrm{d} v .
$$

However, we shall make the stronger requirement, represented by eqn (11). 
Equation (9) can be rewritten in the form

$$
\overline{\boldsymbol{\epsilon}}\left(\theta, \mathbf{A}_{1}\right)-\overline{\boldsymbol{\epsilon}}(\theta, 0)=\left(\frac{\alpha_{1}(\theta)}{4 \rho}-\frac{\theta}{4 \rho} \frac{\mathrm{d} \alpha_{1}(\theta)}{\mathrm{d} \theta}\right)\left|\mathbf{A}_{1}\right|^{2}
$$

We observe that inequality (11), eqn (12), and the fact that $\rho \geq 0$ and $\left|A_{1}\right|^{2} \geq 0$ imply that

$$
\alpha_{1}(\theta)-\theta \frac{\mathrm{d} \alpha_{1}(\theta)}{\mathrm{d} \theta} \geq 0
$$

We are now in a position to make the following assertions.

\section{Assertion 2}

Let an incompressible homogeneous fluid of second grade be compatible with thermodynamics in the sense that all arbitrary motions of the fluid meet the Clausius-Duhem inequality. Further, suppose the assumption that the specific internal energy is a minimum in equilibrium is met. If the material moduli of the fluid are assumed to be constant, then

$$
\alpha_{1} \geq 0 \text {. }
$$

Proof. This result follows trivially from the hypothesis and inequality (13).

\section{Assertion 3}

A necessary and sufficient condition for the specific internal energy of a thermodynamically compatible incompressible second grade fluid to meet assumption (11) is that

$$
\frac{\mathrm{d} \alpha_{1}(\theta)}{\mathrm{d} \theta} \leq \frac{\alpha_{1}(\theta)}{\theta}
$$

Furthermore, a sufficient condition is that

$$
\alpha_{1}(\theta) \geq 0 \text { and } \frac{\mathrm{d} \alpha_{1}(\theta)}{\mathrm{d} \theta} \leq 0
$$

Proof. Follows trivially from inequality (13).

\section{Assertion 4}

Let the material modulus $\alpha_{1}(\theta)$ of a thermodynamically compatible homogeneous second grade fluid be non-positive. Thus a necessary and sufficient condition for assumption (11) to be met is that $\mathrm{d} \alpha_{i}(\theta) / \mathrm{d} \theta$ be non-positive and satisfy the condition

$$
\frac{\mathrm{d} \alpha_{1}(\theta)}{\mathrm{d} \theta} \leq-\frac{\left|\alpha_{1}(\theta)\right|}{\theta} \leq 0
$$

Proof. The proof follows from inequality (13), and the hypothesis that $\theta>0$ and that $\alpha_{1}(\theta) \leq 0$.

While the condition that $\alpha_{1}(\theta) \geq 0$ is both necessary and sufficient for thermodynamically compatible homogeneous fluids of second grade to meet the assumption that the specific Helmholtz free energy be a minimum when the fluid is locally at rest, we can see that the requirement on the specific internal energy leads to a much weaker result regarding $\alpha_{1}(\theta)$.

The Assertions 3 and 4 emphasize the following interesting consequences of requiring the specific internal energy to be a minimum when the fluid is locally at rest. Let us consider the implications of Assertion 3 first. The condition expressed by eqn (16) states that if the normal stress modulus $\alpha_{1}$ is positive and in addition if it decreases with increasing temperature, then the fluid meets the requirements of assumption (11). This feature in the model is in agreement with the physical expectation that the non-Newtonian nature of the fluid becomes less 
significant with increasing temperature. The Assertion 4 indicates that if $\alpha_{1}(\theta) \leq 0$ the normal stress coefficient becomes more negative with increasing temperature, i.e. the non-Newtonian nature of the fluid becomes more significant-a phenomenon which is contrary to physical expectation. These results suggest that the normal stress moduli ought not to be assumed as non-positive for all temperatures. Of course, inequalities (15) and (17) do not preclude the possibility of the normal stress modulus having different signs at different ranges of temperatures. Finally, it is useful to investigate the consequences of the following:

\section{Assumption 2}

The specific entropy of the fluid is a maximum when the fluid is locally at rest, i.e.

$$
\bar{\eta}(\theta, 0) \geq \bar{\eta}\left(\theta, \mathbf{A}_{1}\right)
$$

for all tensors $\mathbf{A}_{1}$ which are traceless.

It follows from eqns $(1)_{2}$ and (5) that

$$
\bar{\eta}\left(\theta, \mathbf{A}_{1}\right)=-\bar{\psi}_{\theta}(\theta, 0)-\frac{1}{4 \rho} \frac{\mathrm{d} \alpha_{1}(\theta)}{\mathrm{d} \theta}\left|\mathbf{A}_{1}\right|^{2} .
$$

Since

$$
\bar{\eta}(\theta, 0)=-\bar{\psi}_{\theta}(\theta, 0)
$$

it follows that

$$
\bar{\eta}\left(\theta, \mathbf{A}_{1}\right)-\bar{\eta}(\theta, 0)=-\frac{1}{4 \rho} \frac{\mathrm{d} \alpha_{1}(\theta)}{\mathrm{d} \theta}\left|\mathbf{A}_{1}\right|^{2}
$$

\section{Assertion 5}

If the specific entropy of a thermodynamically compatible homogeneous incompressible second grade fluid is to meet assumption (18), then

$$
\frac{\mathrm{d} \alpha_{1}}{\mathrm{~d} \theta}(\theta) \geq 0 .
$$

Proof. Inequality (18) can be written as

$$
\bar{\eta}\left(\theta, \mathbf{A}_{1}\right)-\bar{\eta}(\theta, 0) \leq 0
$$

This proof now follows from the inequality and eqn (21).

If assumption (18) is acceptable, then the normal stress effect is enhanced with increasing temperature if $\alpha_{1} \geq 0$, which is physically unacceptable. Of course, Assumptions 1 and 2 together imply that $\alpha_{1}$ cannot be non-positive.

In conclusion, analyses regarding the stability characteristics of second grade fluids wherein the normal stress moduli are dependent on temperature, similar to those carried out in [1], have not been made. It would be interesting to determine whether disturbances in a second grade fluid whose material moduli meet the restrictions imposed by eqns $(1)_{1}$ and (16), decay to the null flow. This, however, is a very complicated problem.

\section{REFERENCES}

[1] J. E. DUNN and R. L. FOSDICK, Arch. Ratl. Mech. Anal. 56, 191 (1974).

12] K. R. RAJAGOPAL, Proc. 16th Midwestern Mech. Conf. 10, 149 (1979).

[3] T. W. TING, Arch. Ratl. Mech. Anal. 14, 1 (1963).

[4] B. D. COLEMAN and H. MARKOVITZ, J. Appl. Phys. 35, 1 (1964).

[5] B. D. COLEMAN, R. J. DUFFIN and V. MIZEL, Arch. Ratl. Mech. Anal. 19, 100 (1965).

[6] B. D. COLEMAN and V. MIZEL, ZAMM 46, 445 (1966).

[7] C. TRUESDELL, Phys. Fluids 8, 1936 (1965).

[8] R. L. FOSDICK and K. R. RAJAGOPAL, Arch. Ratl. Mech. Anal. 70, 145 (1979).

[9] R. S. RIVLIN and J. L. ERICKSEN, J. Ratl. Mech. Anal. 4, 323 (1955). 